\title{
Abnormal corpus callosum induced by overt hepatic encephalopathy impairs interhemispheric functional coordination in cirrhosis patients
}

\author{
Yangying Kuang ${ }^{1}$, Xiaojia Wu ${ }^{2}$, Hua Lai ${ }^{1}$, Zhigang Wang ${ }^{1}$, Qiang Lei ${ }^{1}$, Weijia Zhong ${ }^{2}$, Ya Yang ${ }^{1}$, \\ Chen Deng ${ }^{1}$, Zhiming Zhou ${ }^{2}$
}

${ }^{1}$ Department of Radiology, Chengdu Women's and Children's Central Hospital, School of Medicine, University of Electronic Science and Technology of China, Chengdu, China; ${ }^{2}$ Department of Radiology, The Second Affiliated Hospital of Chongqing Medical University, Chongqing, China

Contributions: (I) Conception and design: Y Kuang; (II) Administrative support: Z Zhou, H Lai, Z Wang; (III) Provision of study materials or patients: All authors; (IV) Collection and assembly of data: Y Kuang, Z Zhou, X Wu; (V) Data analysis and interpretation: Y Kuang, Z Zhou, X Wu; (VI) Manuscript writing: All authors; (VII) Final approval of manuscript: All authors.

Correspondence to: Zhiming Zhou. Department of Radiology, The Second Affiliated Hospital of Chongqing Medical University, 74 Linjiang Road, Chongqing 400010, China. Email: 110039063@qq.com.

Background: Although overt hepatic encephalopathy (OHE) patients were shown to have bilaterally symmetrical structural and functional abnormalities in the whole brain, few studies have focused on the bilateral cerebral hemisphere commissural fibers and measured functional coordination between bilateral hemispheres. This study aimed to investigate the structural changes of the corpus callosum (CC) and interhemispheric functional coordination in patients with $\mathrm{OHE}$ and to test the hypothesis that abnormal CC induced by $\mathrm{OHE}$ impairs interhemispheric functional coordination in cirrhosis patients.

Methods: The microstructural integrity and the volumes of each subregion of the CC were analyzed by diffusion tensor imaging (DTI) and three-dimensional T1-weighted imaging. Voxel-mirrored homotopic connectivity (VMHC) was derived from resting-state functional magnetic resonance imaging (MRI).

Results: Compared with the healthy controls (HCs) and patients without hepatic encephalopathy (noHE), the OHE group showed decreased volumes in all subregions of the CC. In OHE patients, the decreased fractional anisotropy (FA) of CC-5 correlated with decreased VMHC in the middle occipital gyrus (MOG) and precuneus. The value of FA in CC-5 and the volumes of CC-3, CC-4, and CC-5 showed correlations with neuropsychological performance in patients with $\mathrm{OHE}$.

Conclusions: These findings suggest that impairment of interhemispheric white matter pathways may disturb the functional connectivity associated with coordination and neurocognitive performance.

Keywords: Corpus callosum (CC); overt hepatic encephalopathy (OHE); diffusion tensor imaging (DTI); voxelbased morphometry (VBM); voxel-mirrored homotopic connectivity (VMHC)

Submitted Aug 19, 2021. Accepted for publication Oct 21, 2021.

doi: 10.21037/atm-21-5109

View this article at: https://dx.doi.org/10.21037/atm-21-5109

\section{Introduction}

Hepatic encephalopathy (HE), which is caused by hepatic dysfunction in both acute and chronic hepatic diseases, is associated with a wide range of subtle neuropsychiatric disorders and recognizable clinical manifestations, and ranges from minimal HE (MHE) to overt HE (OHE) (1). The estimated incidence of hepatic encephalopathy is 11.6 per 100 person-years, rises to $40 \%$ by 5 years and is accompanied by a survival $<40 \%$ by 12 months after the development of hepatic encephalopathy (2). At the 
most serious stage of this disease, OHE patients exhibit a spectrum of neurocognitive dysfunctions, such as intellectual impairment, personality changes, and consciousness disorders, which are significantly associated with an increased risk of death and a decrease in health-related quality of life $(3,4)$. However, the mechanisms underlying these brain dysfunctions in $\mathrm{OHE}$ are unclear.

Magnetic resonance imaging (MRI) has played an important role in detecting brain abnormalities in OHE patients. Investigations of whole-brain structural changes in OHE patients using voxel-based morphometry (VBM) methods have revealed a bilaterally symmetrical decrease in gray matter (GM) volume (5). Furthermore, decreased GM volume, which indicates structural atrophy, continued to persist after clinical resolution of OHE (6). Diffusion tensor imaging (DTI) can provide a deeper understanding of brain microstructure changes caused by various diseases. Studies have used DTI technology to find that neurocognitive impairment and motor control dysfunction in patients with liver cirrhosis are related to abnormal brain morphology caused by liver cirrhosis (7). Regarding functional changes in OHE, reduced resting-state functional connectivity (FC) within the default mode network (DMN) (7) and widespread changes in FC density across the whole brain have been observed (8), which were associated with neurocognitive impairments in patients. However, despite these bilaterally symmetrical structural and functional abnormalities in the whole brain in OHE, few studies have focused on the bilateral cerebral hemisphere commissural fibers and measured functional coordination between bilateral hemispheres using the voxel-mirrored homotopic connectivity (VMHC) method in patients with OHE. VMHC is a valuable method of resting-state functional MRI (fMRI) used to quantify the resting-state FC between each voxel in one hemisphere and its mirrored counterpart in the other, mainly reflecting the communication pattern between 2 cerebral hemispheres (9). A number of studies have already demonstrated that VMHC is a reliable and reproducible resting-state fMRI metric, and VMHC has already been used to investigate connections between brain hemispheres in schizophrenia, Alzheimer's disease, or primary insomnia (10-12).

The corpus callosum (CC), as the main commissural fiber structure in the brain, plays an important role in $\mathrm{FC}$ between the 2 hemispheres. Abnormal CC structure can impact interhemispheric coordination and communication. In studies examining whole-brain structural changes in MHE using the diffusion tensor imaging (DTI) method, abnormal values of mean diffusivity (MD) and/or fractional anisotropy (FA) were found in the CC $(13,14)$. Furthermore, a study (15) focused on the CC found similar results only in the CC genu and CC splenium, which may provide additional details on structural abnormalities of CC subregions. However, at more serious stages beyond MHE, abnormalities of CC subregions in OHE have not received much attention. In addition, our partition method, based on DTI-based fiber tractography in healthy human subjects as described by Hofer and Frahm (16), suggests that modification of the widely accepted Witelson scheme of defining CC partitions based on primate data is warranted (17).

In this study, we put forward the following hypotheses: (I) OHE patients had structural abnormalities in the subregions of the CC compared with cirrhosis patients without hepatic encephalopathy (noHE) and healthy controls (HCs); (II) abnormalities in subregions of the CC induced by OHE impair interhemispheric functional coordination in cirrhosis patients; (III) there were correlations among structural abnormalities of CC subregions, interhemispheric FC, and neuropsychological tests. To test our hypotheses, we combined 3 methods, including VBM, DTI, and VMHC, to investigate structural and functional abnormalities and identify differences between $\mathrm{OHE}$, noHE, and HC patients. Additionally, Spearman or Pearson correlation analyses were performed to examine the relationships between these structural and functional alterations, neurocognitive performance, and venous ammonia levels. We present the following article in accordance with the MDAR reporting checklist (available at https://dx.doi.org/10.21037/atm-215109).

\section{Methods}

\section{Participants}

All procedures performed in this study involving human participants were in accordance with the Declaration of Helsinki (as revised in 2013). The study was approved by institutional board of the Second Affiliated Hospital of Chongqing Medical University (No. [2019] 018) and informed consent was taken from all the patients. A total of 29 patients with a first episode of OHE and 32 noHE patients were recruited from 2015 to 2018. Each patient underwent laboratory testing of venous ammonia within the first $24 \mathrm{~h}$ in hospital and an MRI evaluation and neuropsychological assessment within 3 days after 
diagnosis of OHE or noHE. The neuropsychological tests included the number connection test A (NCT-A) and digit-symbol test (DST). The inclusion criteria for noHE patients were as follows: firstly, non-alcoholic liver cirrhosis was diagnosed according to clinical criteria, including physical, laboratory, and imaging examination results, and secondly, at least 1 of 2 neuropsychological test results was normal according to the final report of the working party of 11th World Congress of Gastroenterology in Vienna in 1998 (18). Abnormal neuropsychological test results were defined as results exceeding the reference value by 2 standard deviations according to the normal values found for 120 healthy volunteers (18). Non-alcoholic liver cirrhosis patients with a history of OHE were recruited and graded according to the West Haven criteria (19). Additional inclusion criteria for patients were post-hepatitis liver cirrhosis without a recent alcohol history (at least 3 months), and no other diseases or surgery that would affect $\mathrm{HE}$ assessment, such as portosystemic shunt surgery, hepatocellular carcinoma, and other neuropsychological disorders. Subjects were excluded from the study for the following reasons: they were aged $\leq 18$ or $\geq 75$ years; they were not willing to cooperate with the neuropsychological tests; subjects had MRI contraindications; there was head motion of more than $1.0 \mathrm{~mm}$ in translation or greater than $1.0^{\circ}$ rotation during the MRI. Finally, after excluding 3 OHE patients with significant head movement and $1 \mathrm{noHE}$ patient with intracranial subdural hematoma, a total of 26 OHE patients and 31 noHE patients were included in the current study.

Thirty HCs matched for age, sex, and education were enrolled through advertising within the hospital and in nearby communities. None of the HCs had diseases of the liver (cirrhosis, hepatitis, or liver tumors) or neurological/psychiatric disorders. All subjects underwent neuropsychological assessment before cerebral MRI was performed. No laboratory tests were performed in the HCs.

\section{MRI data acquisition}

MRI was performed using a $3.0 \mathrm{~T}$ MR scanner (Achieva Intera, Phillips Medical System) with an 8-channel head coil. All the participants were asked to relax with their eyes closed and their heads motionless without thinking about anything. We used earplugs and fixed each participant's head with sponges of various thicknesses. The MRI data included DTI, three-dimensional $\mathrm{T}_{1}$-weighted imaging $\left(3 \mathrm{DT}_{1}\right)$, blood oxygenation level-dependent (BOLD) sequences, and conventional sequences (axial $\mathrm{T}_{1}$-weighted imaging, $\mathrm{T}_{2}$-weighted imaging, the $\mathrm{T}_{2}$-weighted fluid-attenuated inversion recovery sequence, and sagittal $\mathrm{T}_{1}$-weighted imaging, which were used to exclude patients based on neurological diseases). The DTI data were obtained using a spin echo-based echo-planar imaging sequence [repetition time $(\mathrm{TR})=6,973 \mathrm{~ms}$, echo time $(\mathrm{TE})=75 \mathrm{~ms}$, flip angle $=90^{\circ}$, field of view $(\mathrm{FOV})=224 \times 224 \mathrm{~mm}^{2}$, matrix $=112 \times 112$, and voxel size $\left.=2 \times 2 \times 2 \mathrm{~mm}^{3}\right]$ in contiguous axial planes that included 16 volumes with diffusion gradients applied along 15 noncollinear directions $\left(b=800 \mathrm{~s} / \mathrm{mm}^{2}\right)$ and one volume without diffusion weighting $\left(b=0 \mathrm{~s} / \mathrm{mm}^{2}\right)$. Each volume consisted of 69 contiguous axial slices covering the whole brain. Additionally, $3 \mathrm{DT}_{1}$ was performed in the sagittal orientation using a fast field echo sequence (TR $=7.4 \mathrm{~ms}, \mathrm{TE}=3.6 \mathrm{~ms}$, flip angle $=8^{\circ}, \mathrm{FOV}=250 \times 250 \mathrm{~mm}^{2}$, matrix $=228 \times 227$, slice $=150$, slice thickness $=1.1 \mathrm{~mm}$ with no gap, and voxel size $\left.=1.1 \times 1.1 \times 1.1 \mathrm{~mm}^{3}\right)$. BOLD images were collected using an echo-planar imaging sequence (TR $=2,000 \mathrm{~ms}, \mathrm{TE}=30 \mathrm{~ms}$, flip angle $=90^{\circ}$, matrix $=64 \times 64$, FOV $=240 \times 240 \mathrm{~mm}^{2}$, section thickness $=4.0 \mathrm{~mm}$, slices $=34$, dynamics $=180$ ).

\section{Data reconstruction and processing}

Data processing for BOLD and $3 \mathrm{DT}_{1}$ scans was performed using the Data Processing Assistant for Resting-State fMRI (DPARSF) toolbox (20), REST tools http://www. restfmri.net/forum/REST_V1.8), and Statistical Parametric Mapping (SPM) 8 software (www.fil.ion.ucl.ac.uk/spm/ software/spm8/) for MATLAB, and DTI data processing was conducted using FSL tools (https://fsl.fmrib.ox.ac. $\mathrm{uk} / \mathrm{fsl} / \mathrm{fs} l w i k i /)$. The main steps of data processing are as follows.

The $3 \mathrm{D}$ masks of CC subregions were produced by a senior neuroradiologist based on the FMRIB58 template using MRIcron software. First, the boundaries of the CC were determined as follows: the superior, anterior, and posterior sides constituted the callosal sulcus and the cingulate gyrus; the inferior side was bounded by the ventricular surface; and the lateral side was bounded by a straight line attaching the callosal sulcus and the lateralsuperior corner of the caudate nucleus or lateral ventricle. Second, 5 segmentations were determined according to the scheme proposed by Hofer and Frahm (16). Third, each 3D mask of the CC subregions was manually traced by the 

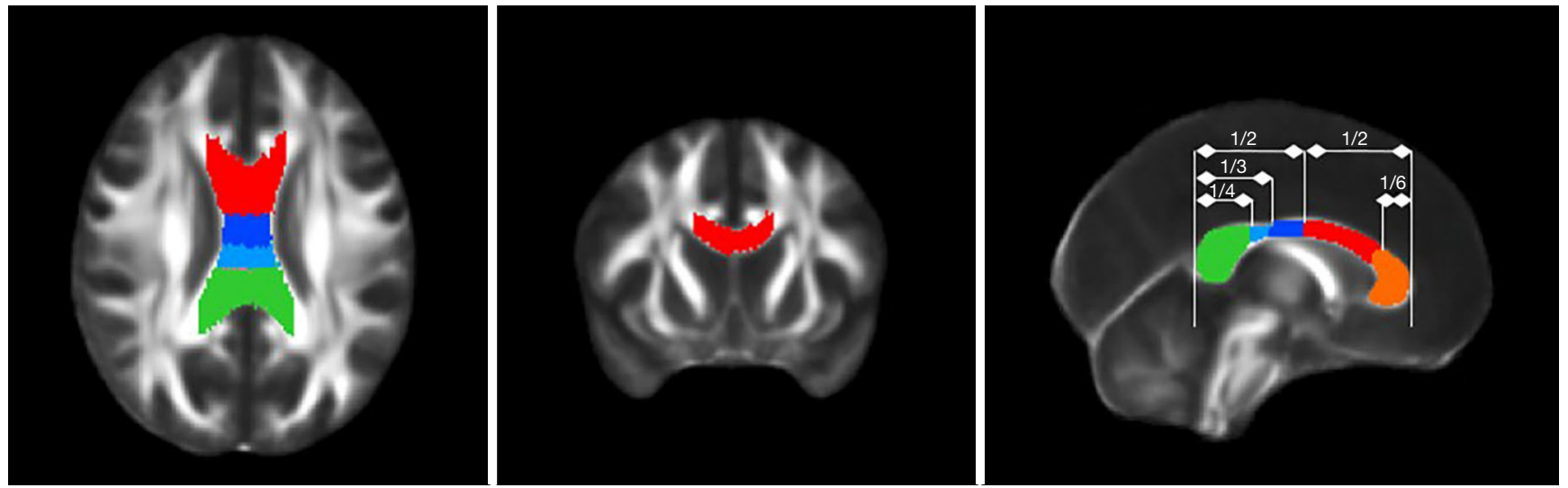

Figure 1 The three-dimensional masks of corpus callosum (CC) subregions on the FMRIB58 template. Six lines were set perpendicular to the anterior-posterior axis. The diagram includes the following 5 subregions: prefrontal (CC-1, orange), premotor/supplementary motor (CC-2, red), primary motor (CC-3, deep blue), primary sensory (CC-4, light blue), and parietal/temporal/visual (CC-5, green).

senior neuroradiologist from the midsagittal plane based on the anatomical boundaries to assess the volume of interest (VOI). The process was repeated 3 times, and the best VOI was selected for analysis (Figure 1). Three senior doctors voted on the optimal VOI based on the degree of matching of the mask to the anatomy.

Preprocessing of the DTI data was performed using FSL to eliminate the distortion induced by head motion and eddy current. Then, non-brain tissue in the images was removed by FSL's Brain Extraction Tool. Finally, FA, MD, axial diffusivity $(\mathrm{AD})$, and radial diffusivity (RD) maps were computed. To ensure the same resolution of the 3D mask, all individual parametric maps were re-registered to the FMRIB58 template, and the parametric value of each CC subregion was extracted using REST software.

DARTEL-based VBM analysis was performed using SPM8 and REST software. First, all $3 \mathrm{DT}_{1}$ scans were corrected for any bias and divided into GM, white matter (WM), and cerebrospinal fluid (CSF) by a unified segmentation model. Second, a GM population template was derived from the entire image dataset using the DARTEL method. Third, after initial affine registration of the DARTEL template to the GM tissue probability map in the Montreal Neurological Institute (MNI) space, nonlinear warping of the segmented images was then performed to match the DARTEL template in the MNI space. Fourth, the modulated volumes were smoothed with an isotropic Gaussian kernel of 6-mm full width at half maximum (FWHM), and the original tissue volume was reestablished prior to spatial normalization. Finally, the WM parameter map and GM parameter map were archived for further analysis. To ensure the same resolution as the $3 \mathrm{D}$ mask, all individual $T_{1}$-weighted image volume data were re-registered to the FMRIB58 template, and the volume of each CC subregion was extracted using REST software for analysis.

BOLD image analyses were performed using the DPARSF toolbox to calculate the VMHC. Data preprocessing included removal of the first 10 time points (for adaption to the scanning noise and the steady state of the machine), slice-timing correction (to correct slicetiming differences), realign correction (to correct slight head movement), spatial normalization (spatially normalized to the MNI space and resampled to a voxel size of $3 \times 3 \times 3 \mathrm{~mm}^{3}$ ), spatial smoothing (smoothed with a Gaussian kernel of 6-mm FWHM), linear detrending and bandpass filtering (with a frequency range of $0.01-0.08 \mathrm{~Hz}$ ), and removal of nuisance signals of WM, CSF, and 6 motion parameters and global signals.

The calculation procedures for VMHC were based on a previous study (7). First, normalized $\mathrm{T}_{1}$-weighted structural images of all subjects were averaged to generate a mean structural image, which was used to create its flipped version. Then, the symmetric $\mathrm{T}_{1}$-weighted image template was obtained by averaging the above mean image with a bilateral mirrored version. Second, normalized individual $\mathrm{T}_{1}$-weighted images were re-normalized to the symmetric $\mathrm{T}_{1}$-weighted image template by nonlinear registration. Next, the above transformation was applied to each participant's preprocessed functional data. Finally, the 
Table 1 Summary of demographic information and neuropsychological test scores for the 3 groups

\begin{tabular}{|c|c|c|c|c|c|}
\hline Variables & OHE $(n=26)$ & noHE $(n=31)$ & $\mathrm{HCs}(\mathrm{n}=30)$ & $\mathrm{F}$ & $\mathrm{P}$ \\
\hline Age (years) & $54.46 \pm 11.00$ & $54.42 \pm 8.83$ & $53.93 \pm 12.47$ & 0.021 & 0.979 \\
\hline Education (years) & $8.54 \pm 3.47$ & $9.39 \pm 3.04$ & $10.13 \pm 4.21$ & 1.364 & 0.261 \\
\hline NCT-A (seconds) & $60.46 \pm 29.96$ & $35.76 \pm 11.05$ & $31.97 \pm 13.54$ & 17.491 & 0.000 \\
\hline $\mathrm{NH}_{3}(\mu \mathrm{mol} / \mathrm{L})$ & $80.03 \pm 45.45$ & $19.58 \pm 12.44$ & & & \\
\hline Child-Pugh (A/B/C) & $4 / 8 / 14$ & $19 / 11 / 1$ & & & \\
\hline
\end{tabular}

OHE, overt hepatic encephalopathy; noHE, cirrhosis without HE; HCs, healthy controls; NCT-A, number connection test A; DST, digitsymbol test.

Pearson correlation between the time series of every pair of symmetrical interhemispheric voxels was calculated to measure the interhemispheric homotopic resting-state FC. The computed correlation coefficients were then Fisher's r-to-z transformed to obtain normalized z-map data, which were regarded as the zVMHC values and were used for statistical analysis.

\section{Statistical analysis}

VMHC differences within the whole brain among the 3 groups were assessed by REST 1.8 software. The statistical threshold was set at $\mathrm{P}<0.05$ for individual voxels corrected using the AFNI AlphaSim program (http://afni.nih.gov/ afni/docpdf/AlphaSim.pdf), and the minimum cluster size was 54 voxels. Statistical analyses of the other data were performed with SPSS 19.0 (SPSS Inc., Chicago, IL, USA), and the threshold of significance was set at a level of $\mathrm{P}<0.05$.

Sex ratios between the 3 groups were analyzed using chisquare tests. Age, education, NCT-A and DST scores, DTI indices, and the volumes of subregions in the CC between the $\mathrm{HC}$, noHE, and OHE groups were analyzed using oneway analysis of variance (ANOVA). One-way analysis of covariance with age and sex as covariates was performed by REST 1.8 software to determine VMHC differences within the whole brain among the 3 groups. Post-boc analyses with LSD corrections identified regions with significant differences in VMHC, DTI indices, and volumes between groups. Spearman or Pearson correlation analyses were used to examine the relationship among blood ammonia levels, neuropsychological test scores, and structural and functional alterations.

\section{Results}

\section{Demographics, neuropsychological test results, and biochemical parameters}

The demographics, neuropsychological test results, and biochemical parameters for all 87 participants are summarized in Table 1. No significant differences were found in terms of age, sex, and education level among the OHE, noHE, and HC groups $(\mathrm{P}>0.05)$. The NCT-A and DST scores significantly differed among the 3 groups $(\mathrm{P}<0.001)$. Compared with the HCs and noHE patients, OHE patients spent more time completing the NCT-A test and had fewer correct responses on the DST.

\section{DTI, VBM, and VMHC results}

The ANOVA results showed that the volumes of all CC subregions and the FA values in CC-5 were significantly different among the 3 groups. Compared with the HC group, the $\mathrm{OHE}$ and noHE groups exhibited decreased FA in CC-5 $(\mathrm{P}<0.05)$. No significant differences were found in the $\mathrm{MD}, \mathrm{AD}$, or $\mathrm{RD}$ in all $\mathrm{CC}$ subregions among the 3 groups. Compared with the other 2 groups, the $\mathrm{OHE}$ group exhibited decreased volumes in all subregions of the CC (Table 2). The bilateral middle occipital gyrus (MOG), bilateral precentral gyrus, bilateral middle temporal gyrus (MTG), frontal lobe, and bilateral precuneus showed decreased VMHC values in both the OHE patients (Figure 2A) and the noHE patients (Figure 2B) compared with the corresponding values in the HCs. Compared with the noHE group, the OHE group showed increased VMHC values in the bilateral MOG (Figure 2C). 
Table 2 Subregions of the CC showing significant differences in DTI indices and volume between the OHE, noHE, and HC groups

\begin{tabular}{lccccccc}
\hline Indices & Region & OHE $(\mathrm{n}=26)$ & $\operatorname{noHE}(\mathrm{n}=31)$ & $\mathrm{HCs}(\mathrm{n}=30)$ & $\mathrm{F}$ & $\mathrm{P}$ & Post hoc comparison \\
\hline FA & CC-5 & $0.49223 \pm 0.06658$ & $0.45925 \pm 0.09463$ & $0.54490 \pm 0.06856$ & 9.228 & 0.000 & $\mathrm{OHE}<\mathrm{HC}, \mathrm{noHE}<\mathrm{HC}$ \\
Volume $\left(\mathrm{cm}^{3}\right)$ & CC-1 & $0.83300 \pm 0.08516$ & $0.88577 \pm 0.01617$ & $0.88886 \pm 0.01689$ & 11.461 & 0.000 & $\mathrm{OHE}<\mathrm{HC}, \mathrm{OHE}<\mathrm{noHE}$ \\
& CC-2 & $0.80792 \pm 0.09702$ & $0.85690 \pm 0.02350$ & $0.85866 \pm 0.02120$ & 7.188 & 0.001 & $\mathrm{OHE}<\mathrm{HC}, \mathrm{OHE}<\mathrm{noHE}$ \\
& CC-3 & $0.79873 \pm 0.14470$ & $0.85664 \pm 0.03194$ & $0.85710 \pm 0.02883$ & 4.476 & 0.014 & $\mathrm{OHE}<\mathrm{HC}, \mathrm{OHE}<\mathrm{noHE}$ \\
& CC-4 & $0.78635 \pm 0.16055$ & $0.84603 \pm 0.04083$ & $0.84590 \pm 0.03589$ & 3.718 & 0.028 & $\mathrm{OHE}<\mathrm{HC}, \mathrm{OHE}<\mathrm{noHE}$ \\
& CC-5 & $0.80034 \pm 0.07107$ & $0.85096 \pm 0.01405$ & $0.84860 \pm 0.01371$ & 13.629 & 0.000 & $\mathrm{OHE}<\mathrm{HC}, \mathrm{OHE}<\mathrm{noHE}$ \\
\end{tabular}

DTI, diffusion tensor imaging; OHE, overt hepatic encephalopathy; noHE, cirrhosis without HE; HCs, healthy controls; CC, corpus callosum; FA, fractional anisotropy.

Correlations among structural abnormalities in subregions of the CC, interhemispheric connectivity, blood ammonia levels, and neuropsychological test scores in OHE patients

The correlations among DTI indices, volumes, and neuropsychological test scores in OHE patients are shown in Tables 3,4. The FA values in CC-5 had positive correlations with the $\mathrm{VMHC}$ values in the bilateral precuneus $(\mathrm{r}=0.785, \mathrm{P}<0.001)$ and bilateral MOG $(\mathrm{r}=0.489$, $\mathrm{P}=0.001$ ) (Figure 3).

\section{Discussion}

In the present study, our results demonstrated volume or FA changes in CC subregions and decreased VMHC in some regions of the parietal and occipital lobes in OHE patients, suggesting that impairment of interhemispheric WM pathways may disturb the FC associated with coordination and neurocognitive performance.

\section{Abnormal structure within the CC in OHE patients}

For structural changes of the CC, compared with the HC and noHE groups, the OHE group showed decreased volumes in each CC subregion, supporting the notion that macrostructure atrophy occurs in the CC in OHE patients. The pathological death of neuronal cells (21) and the development of WM atrophy (22) have been sufficiently confirmed in HE patients. Moreover, considering the important role of the WM in transmitting instructions, WM atrophy, which may be induced by the death of neuronal cells, may contribute to brain dysfunction. For example, a previous study (23) found relationships between WM atrophy and neurocognitive impairments in cirrhosis patients and speculated that WM atrophy may be one of the mechanisms underlying neurocognitive deficits. The present study revealed that the decreased volumes in CC-3, CC-4, and CC-5 were also significantly associated with the poor neurocognitive performance of $\mathrm{OHE}$ patients. The CC-3, CC-4, and CC-5, affecting connections to the bilateral motor areas, sensory areas, and parietal, temporal, and occipital gyri, participate in motor, sensory, working memory, and visual processes. Ye and colleagues (24) found that the CC-3 volume of patients with liver cirrhosis (minimal hepatic encephalopathy and no minimal hepatic encephalopathy) was significantly positively correlated with the PHES score. We speculate that this may be related to the course of the disease. As the disease progresses, the CC-4 and CC-5 volumes may change more significantly.

The FA value reflects the proportion of the anisotropic components of water molecules in the overall diffusion tensor. The decrease of the FA value indirectly indicates the destruction of the integrity of the white matter fiber bundles, and there may be loss of myelin sheath, axonal damage, and abnormal oligodendrocyte proliferation (25). Our results of decreased FA values in the CC-5 of OHE patients compared to the HCs suggests a role of microstructural integrity damage in OHE patients. Although the neuropathological mechanisms of decreased FA are not well understood in HE, one possible explanation is axon loss (26). Therefore, we speculated that the decreased FA in CC-5 may represent impaired axons secondary to the coincident loss of neurons. We did not detect any significant changes in $\mathrm{MD}, \mathrm{AD}$, or $\mathrm{RD}$ in all $\mathrm{CC}$ subregions among the 3 groups. This neuroimaging study of cirrhosis may also be limited by the relatively small sample size. Future studies with larger sample size are needed to confirm and better delineate the neuroimaging results. Taken together, these data suggest that macrostructure atrophy of all CC 
A
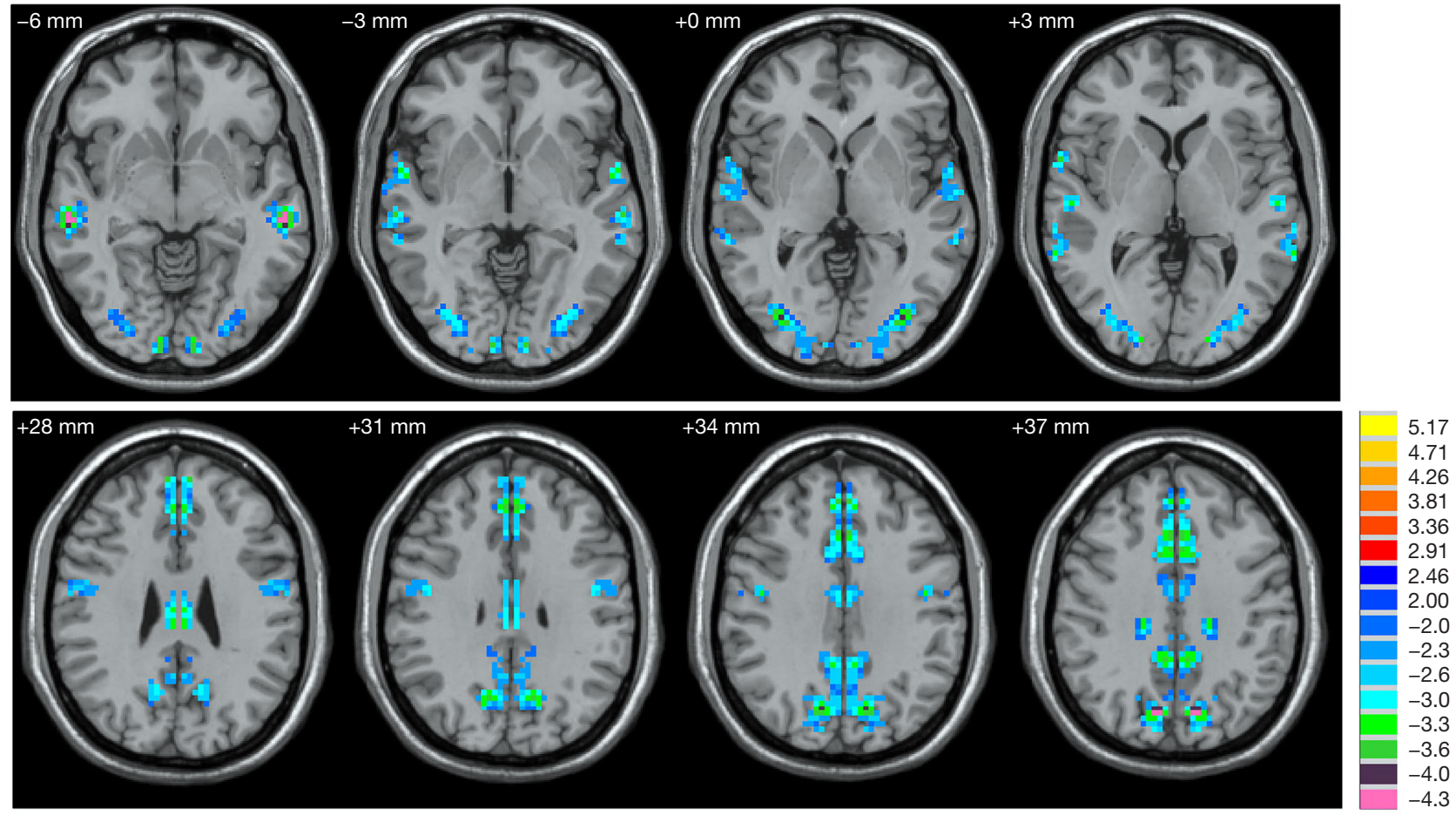

B
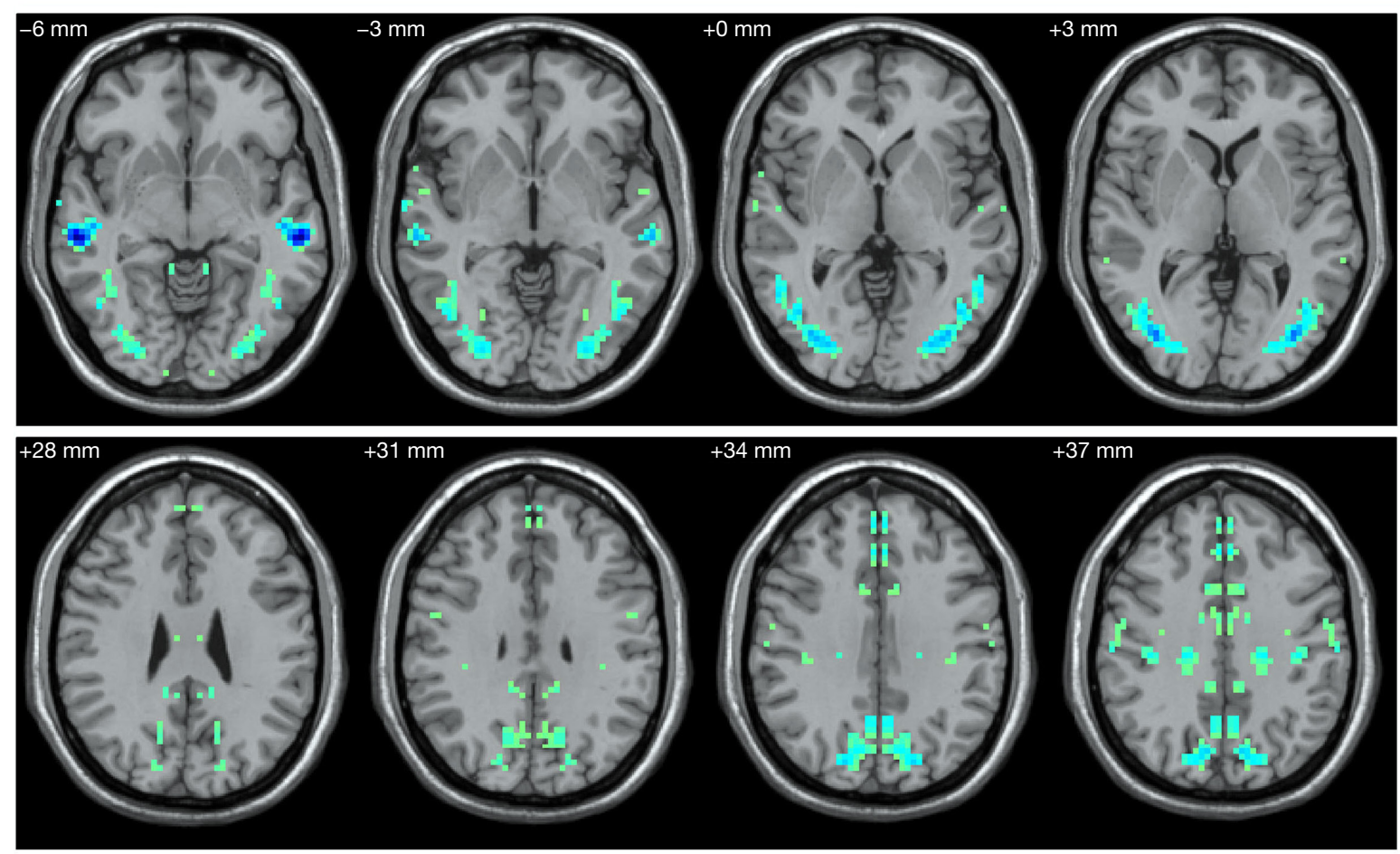

$-2.00$ 


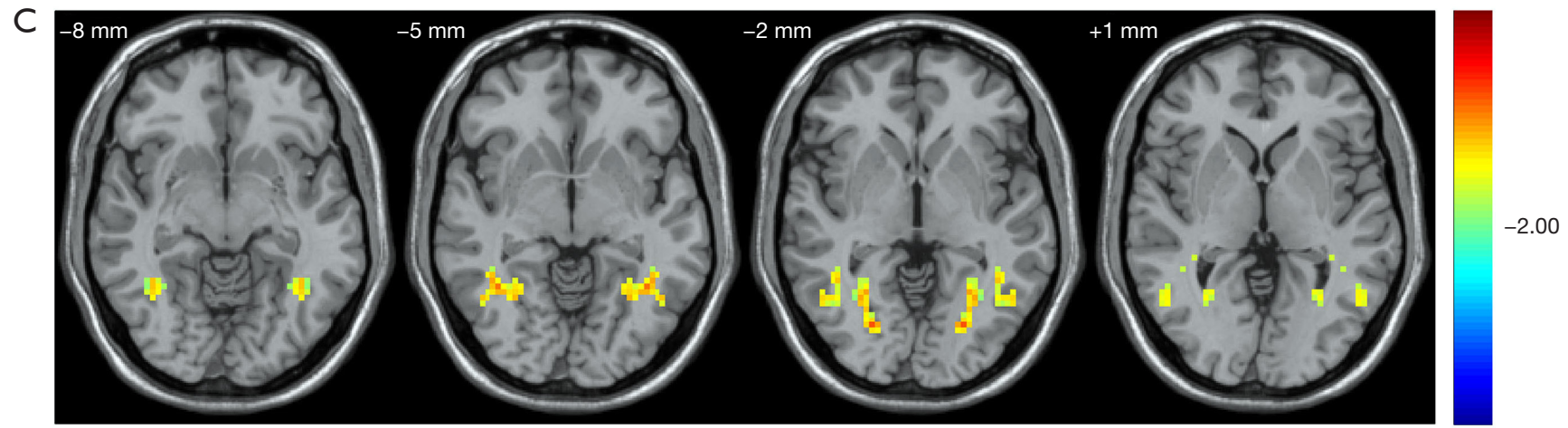

Figure 2 The brain regions with between-group differences in voxel-mirrored homotopic connectivity (VMHC). (A) The bilateral middle occipital gyrus (MOG), bilateral precentral gyrus, bilateral middle temporal gyrus (MTG), frontal lobe, and bilateral precuneus showing significantly decreased VMHC in overt hepatic encephalopathy (OHE) patients compared with the healthy controls (HCs) (regions highlighted in blue color). (B) The bilateral MOG, bilateral precentral gyrus, bilateral MTG, frontal lobe, and bilateral precuneus showing significantly decreased VMHC in cirrhosis patients without hepatic encephalopathy (noHE) compared with the HCs (regions highlighted in blue color). (C) Compared with the noHE group, the OHE group showed increased VMHC values in the bilateral MOG (regions highlighted in yellow color).

Table 3 Subregions of the CC showing significant correlations between DTI indices and neuropsychological test scores in the OHE group

\begin{tabular}{lccc}
\hline $\begin{array}{l}\text { Neuropsychological } \\
\text { tests }\end{array}$ & DTI indices & Correlation $(r)$ & $P$ \\
\hline NCT-A & FA (CC-5) & -0.392 & 0.048 \\
DST & FA (CC-5) & 0.515 & 0.007 \\
\hline
\end{tabular}

DTI, diffusion tensor imaging; OHE, overt hepatic encephalopathy; CC, corpus callosum; FA, fractional anisotropy; NCT-A, number connection test A; DST, digit-symbol test.

subregions and microstructural impairment of CC-5 may constitute the basis of neurocognitive dysfunction in $\mathrm{OHE}$.

\section{Abnormal VMHC in OHE patients}

In our study, compared with the HC group, VMHC values in the bilateral precuneus were decreased in the $\mathrm{OHE}$ group. As an important hub of the DMN, the precuneus participates in large-scale supervision of integrated processes, including working memory, voluntary attention shifts, and visuospatial integration (27). In a previous study (7) using independent component analysis to identify the best-fit components for the DMN in different stages of $\mathrm{HE}$, the dominant reduction in $\mathrm{FC}$ within the DMN including the bilateral precuneus was observed in
Table 4 Subregions of the CC showing significant correlations between volume and neuropsychological test scores in the OHE group

\begin{tabular}{lccc}
\hline $\begin{array}{l}\text { Neuropsychological } \\
\text { tests }\end{array}$ & Volume & Correlation $(r)$ & $P$ \\
\hline NCT-A & CC-3 & -0.546 & 0.004 \\
DST & CC-3 & 0.541 & 0.004 \\
NCT-A & CC-4 & -0.457 & 0.019 \\
DST & CC-4 & 0.447 & 0.022 \\
NCT-A & CC-5 & -0.454 & 0.020 \\
DST & CC-5 & 0.448 & 0.022 \\
\hline
\end{tabular}

DTI, diffusion tensor imaging; OHE, overt hepatic encephalopathy; CC, corpus callosum; NCT-A, number connection test A; DST, digit-symbol test.

cirrhotic patients with current MHE and previous OHE. This suggests that FC within the DMN can become a supplementary imaging marker to evaluate the cognitive status of cirrhotic patients with latent OHE. Thus, using VMHC analysis, we speculated that the FC between the bilateral precuneus may impact the DMN, thus leading to dysfunction of the supervision and integration of these processes in OHE patients. In addition to the precuneus, compared with the HCs, OHE patients showed decreased VMHC values within the bilateral MOG and bilateral 
A

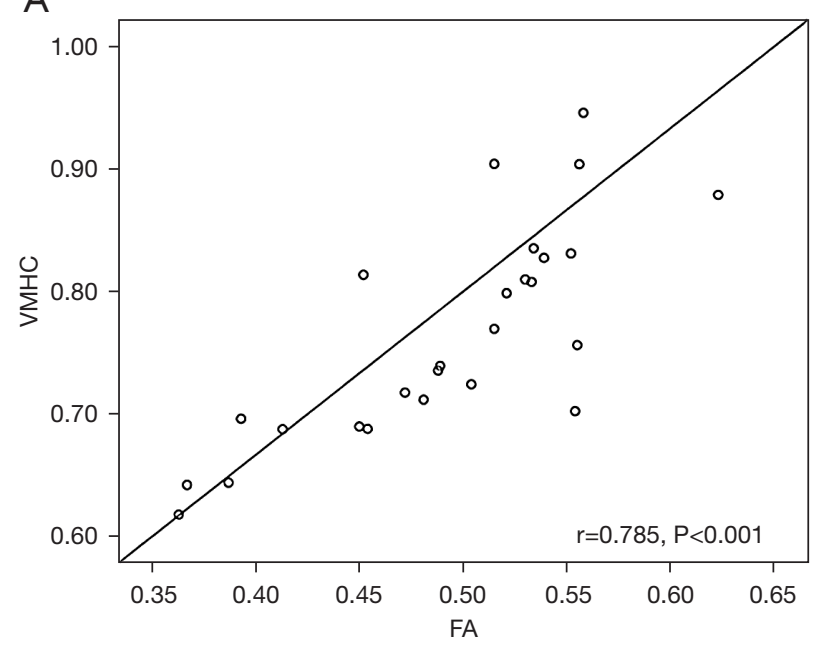

B

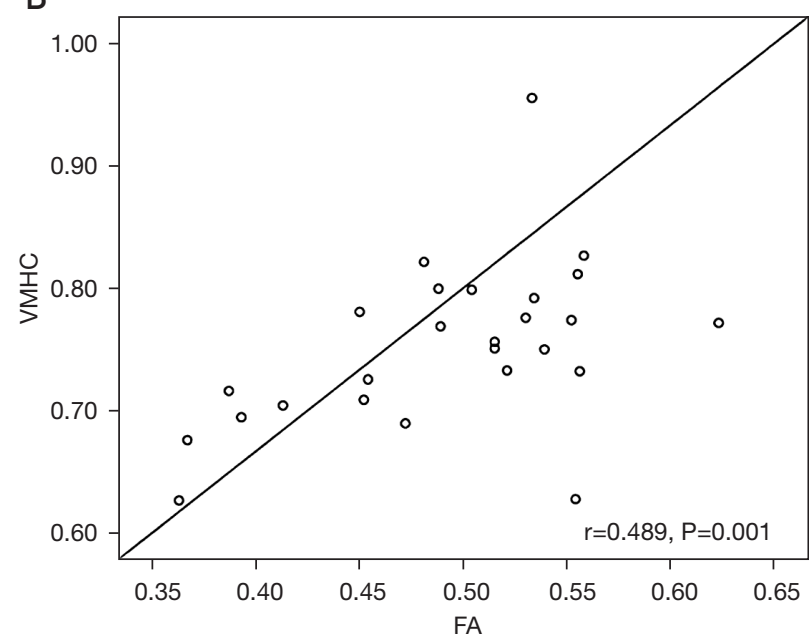

Figure 3 Pearson correlation results reveal the correlations between fractional anisotropy (FA) in corpus callosum (CC)-5 and voxelmirrored homotopic connectivity (VMHC) in overt hepatic encephalopathy patients. (A) FA in CC-5 shows a positive correlation with VMHC in the bilateral precuneus. (B) FA in CC-5 shows a positive correlation with VMHC in the bilateral middle occipital gyrus.

MTG, which may indicate that the FC between the bilateral MOG and MTG was weaker than that in HCs. The MOG is the secondary visual cortex, which mainly manages the vision-related functional activities of the brain. Ye and colleagues (24) found that minimal hepatic encephalopathy patients had lower VMHC among bilateral inferior parietal lobe, superior temporal gyrus, precentral gyrus, posterior central gyrus, cuneus, lingual gyrus, MOG, MTG regions compared with that of normal controls. We speculate that as the disease progresses, different brain regions have different degrees of self-repair.

Additionally, the MTG is involved in visual attention processes. Similarly, previous findings have suggested that decreased FC in the bilateral MTG was correlated with neuropsychological impairments in HE patients (28). These $3 \mathrm{FC}$ areas between the 2 hemispheres are connected by the fibers that travel through CC-5. In addition, we found that decreased VMHC of the precuneus and MOG were paralleled with decreased FA of CC-5. Therefore, the decreased VMHC of the precuneus and MOG may be caused by the impaired axons of CC-5, thereby promoting working memory and visual dysfunction and subsequent poor performance on the NCT-A and DST. It is worth mentioning that the present work revealed that the structures of the CC subregions had changed, but some of the FC which corresponded to the bilateral cerebral hemispheres did not. This may imply that the structural changes of CC subregions are more obvious than FC between interhemispheric regions.

\section{Conclusions}

In conclusion, structural abnormalities within subregions of the $\mathrm{CC}$ and disrupted interhemispheric $\mathrm{FC}$ are additional characteristics of OHE. Moreover, the structural abnormalities in CC-3, CC-4, and CC-5 are associated with patients' cognitive impairments. Finally, the coordination dysfunction of the bilateral MOG and precuneus may be caused by structural abnormalities of CC-5. These data may improve the current understanding of the neural underpinnings of OHE.

\section{Acknowledgments}

Funding: None.

\section{Footnote}

Reporting Checklist: The authors have completed the MDAR reporting checklist. Available at https://dx.doi. org/10.21037/atm-21-5109

Data Sharing Statement: Available at https://dx.doi. org/10.21037/atm-21-5109 
Conflicts of Interest: All authors have completed the ICMJE uniform disclosure form (available at https://dx.doi. org/10.21037/atm-21-5109). The authors have no conflicts of interest to declare.

Etbical Statement: The authors are accountable for all aspects of the work in ensuring that questions related to the accuracy or integrity of any part of the work are appropriately investigated and resolved. All procedures performed in this study involving human participants were in accordance with the Declaration of Helsinki (as revised in 2013). The study was approved by institutional board of the Second Affiliated Hospital of Chongqing Medical University (No. [2019] 018) and informed consent was taken from all the patients.

Open Access Statement: This is an Open Access article distributed in accordance with the Creative Commons Attribution-NonCommercial-NoDerivs 4.0 International License (CC BY-NC-ND 4.0), which permits the noncommercial replication and distribution of the article with the strict proviso that no changes or edits are made and the original work is properly cited (including links to both the formal publication through the relevant DOI and the license). See: https://creativecommons.org/licenses/by-nc-nd/4.0/.

\section{References}

1. Patidar KR, Bajaj JS. Covert and Overt Hepatic Encephalopathy: Diagnosis and Management. Clin Gastroenterol Hepatol 2015;13:2048-61.

2. Tapper EB, Aberasturi D, Zhao Z, et al. Outcomes after hepatic encephalopathy in population-based cohorts of patients with cirrhosis. Aliment Pharmacol Ther 2020;51:1397-405.

3. Barboza KC, Salinas LM, Sahebjam F, et al. Impact of depressive symptoms and hepatic encephalopathy on health-related quality of life in cirrhotic hepatitis $\mathrm{C}$ patients. Metab Brain Dis 2016;31:869-80.

4. Stewart CA, Malinchoc M, Kim WR, et al. Hepatic encephalopathy as a predictor of survival in patients with end-stage liver disease. Liver Transpl 2007;13:1366-71.

5. Zhang LJ, Qi R, Zhong J, et al. The effect of hepatic encephalopathy, hepatic failure, and portosystemic shunt on brain volume of cirrhotic patients: a voxel-based morphometry study. PLoS One 2012;7:e42824.

6. Chen HJ, Zhu XQ, Shu H, et al. Structural and functional cerebral impairments in cirrhotic patients with a history of overt hepatic encephalopathy. Eur J Radiol 2012;81:2463-9.

7. Chen HJ, Jiao Y, Zhu XQ, et al. Brain dysfunction primarily related to previous overt hepatic encephalopathy compared with minimal hepatic encephalopathy: restingstate functional MR imaging demonstration. Radiology 2013;266:261-70.

8. Zhang G, Cheng Y, Liu B. Abnormalities of voxel-based whole-brain functional connectivity patterns predict the progression of hepatic encephalopathy. Brain Imaging Behav 2017;11:784-96.

9. Zuo XN, Kelly C, Di Martino A, et al. Growing together and growing apart: regional and sex differences in the lifespan developmental trajectories of functional homotopy. J Neurosci 2010;30:15034-43.

10. Liao ZL, Tan YF, Qiu YJ, et al. Interhemispheric functional connectivity for Alzheimer's disease and amnestic mild cognitive impairment based on the triple network model. J Zhejiang Univ Sci B 2018;19:924-34.

11. Dai XJ, Liu BX, Ai S, et al. Altered inter-hemispheric communication of default-mode and visual networks underlie etiology of primary insomnia : Altered interhemispheric communication underlie etiology of insomnia. Brain Imaging Behav 2020;14:1430-44.

12. Hoptman MJ, Zuo XN, D'Angelo D, et al. Decreased interhemispheric coordination in schizophrenia: a resting state fMRI study. Schizophr Res 2012;141:1-7.

13. Kumar R, Gupta RK, Elderkin-Thompson V, et al. Voxelbased diffusion tensor magnetic resonance imaging evaluation of low-grade hepatic encephalopathy. J Magn Reson Imaging 2008;27:1061-8.

14. Qi R, Zhang LJ, Zhong J, et al. Grey and white matter abnormalities in minimal hepatic encephalopathy: a study combining voxel-based morphometry and tract-based spatial statistics. Eur Radiol 2013;23:3370-8.

15. Chen HJ, Wang Y, Yang M, et al. Aberrant interhemispheric functional coordination in patients with HBV-related cirrhosis and minimal hepatic encephalopathy. Metab Brain Dis 2014;29:617-23.

16. Hofer S, Frahm J. Topography of the human corpus callosum revisited--comprehensive fiber tractography using diffusion tensor magnetic resonance imaging. Neuroimage 2006;32:989-94.

17. Witelson SF. Hand and sex differences in the isthmus and genu of the human corpus callosum. A postmortem morphological study. Brain 1989;112:799-835.

18. Ferenci P, Lockwood A, Mullen K, et al. Hepatic encephalopathy--definition, nomenclature, diagnosis, and 
quantification: final report of the working party at the 11th World Congresses of Gastroenterology, Vienna, 1998. Hepatology 2002;35:716-21.

19. Atterbury CE, Maddrey WC, Conn HO. Neomycinsorbitol and lactulose in the treatment of acute portalsystemic encephalopathy. A controlled, double-blind clinical trial. Am J Dig Dis 1978;23:398-406.

20. Chao-Gan Y, Yu-Feng Z. DPARSF: A MATLAB Toolbox for "Pipeline" Data Analysis of Resting-State fMRI. Front Syst Neurosci 2010;4:13.

21. Butterworth R. Neuronal cell death in hepatic encephalopathy. Metab Brain Dis 2007;22:309-20.

22. Guevara M, Baccaro ME, Gómez-Ansón B, et al. Cerebral magnetic resonance imaging reveals marked abnormalities of brain tissue density in patients with cirrhosis without overt hepatic encephalopathy. J Hepatol 2011;55:564-73.

23. Chen HJ, Wang Y, Zhu XQ, et al. White matter abnormalities correlate with neurocognitive performance in patients with $\mathrm{HBV}$-related cirrhosis. J Neurol Sci 2012;321:65-72.

Cite this article as: Kuang Y, Wu X, Lai H, Wang Z, Lei Q, Zhong W, Yang Y, Deng C, Zhou Z. Abnormal corpus callosum induced by overt hepatic encephalopathy impairs interhemispheric functional coordination in cirrhosis patients. Ann Transl Med 2021;9(20):1579. doi: 10.21037/atm-21-5109
24. Ye M, Guo Z, Li Z, et al. Aberrant inter-hemispheric coordination characterizes the progression of minimal hepatic encephalopathy in patients with $\mathrm{HBV}$-related cirrhosis. Neuroimage Clin 2020;25:102175.

25. Wilting J, Rolfsnes HO, Zimmermann H, et al. Structural correlates for fatigue in early relapsing remitting multiple sclerosis. Eur Radiol 2016;26:515-23.

26. Schmierer K, Wheeler-Kingshott CA, Boulby PA, et al. Diffusion tensor imaging of post mortem multiple sclerosis brain. Neuroimage 2007;35:467-77.

27. Cavanna AE, Trimble MR. The precuneus: a review of its functional anatomy and behavioural correlates. Brain 2006;129:564-83.

28. Qi R, Zhang LJ, Luo S, et al. Default mode network functional connectivity: a promising biomarker for diagnosing minimal hepatic encephalopathy: CONSORTcompliant article. Medicine (Baltimore) 2014;93:e227.

(English Language Editor: C. Betlazar-Maseh) 